\title{
DEFICIENCY IN F-MANIFOLDS
}

\author{
WILLIAM H. CUTLER ${ }^{1}$
}

\begin{abstract}
Let $M$ be a manifold modelled on a metrizable, locally-convex, topological vector space $F$ such that $F \cong F^{\omega}$, and let $K$ be a closed subset of $M$. Then the following are equivalent: (1) $K$ is locally a subset of a collared submanifold of $M$, (2) each $x \in K$ has an open neighborhood $U$ and a homeomorphism $h: U \rightarrow l_{2} \times F$ such that $h(U \cap K) \subset\{0\} \times F$, (3) each $x \in K$ has an open neighborhood $U$ and a homeomorphism $h: U \rightarrow F \times F$ such that $h(U \cap K) \subset$ $\{0\} \times F$, (4) there is a homeomorphism $h: M \rightarrow M \times F$ such that for $x \in K, h(x)=(x, 0),(5) K$ is infinite-deficient (i.e. there is a homeomorphism $h: M \rightarrow M \times l_{2}$ such that $\left.h(K) \subset M \times\{0\}\right)$, and (6) $K$ is the finite union of sets each having one of the above properties.
\end{abstract}

1. Introduction. Let $F$ be a metrizable, locally-convex, topological vector space (MLCTVS) such that $F \cong F^{\omega}$ ( $F$ is homeomorphic to the countable product of itself). ${ }^{2}$ An $F$-manifold is a paracompact Hausdorff space with a covering of open sets homeomorphic to $F$. Deficient subsets of $F$-manifolds are essentially those which are contained in subspaces of infinite codimension. Anderson [1] was the first to do work in this area, and he was concerned at that time with separable Fréchet spaces (which are all homeomorphic to $s$, the countable product of lines, and $l_{2}$, separable Hilbert space, see [2]).

Recent results have tied up the notions of deficiency with that of negligibility $(Y \subset X$ is negligible if $X \cong X-Y$ ) and that of Property $\mathrm{Z}$ (homotopic negligibility). (See [3] and [4].) This paper characterizes the concept of deficiency for closed subsets of $F$-manifolds.

Let $M$ be an $F$-manifold and let $K$ be a closed subset of $M . K$ is locally infinite-deficient (1.i.d.) if each point in $K$ has an open neighborhood $U$ and a homeomorphism $h: U \rightarrow F \times l_{2}$ such that $h(U \cap K) \subset F \times\{0\} . K$ is locally $F$-deficient if each point of $K$ has an open neighborhood $U$ and a

Received by the editors April 28, 1971.

AMS 1970 subject classifications. Primary 57A20, 58B05; Secondary 57A20, 58B05.

Key words and phrases. Infinite-dimensional manifold, deficiency, negligibility, variable product.

1 This paper is part of the author's dissertation done under David W. Henderson.

${ }^{2}$ The condition "locally-convex" can be replaced by " $F \cong R \times Z$ for some metric space $Z$ ". The author knows of no MTVS's where this is not true.

(c) American Mathematical Society 1972 
homeomorphism $h: U \rightarrow F \times F$ such that $h(U \cap K) \subset F \times\{0\} . K$ is $F$-deficient if there is a homeomorphism $h: M \rightarrow M \times F$ such that $h(K) \subset M \times\{0\}$. Finally, $K$ is infinite-deficient (as it is usually defined in the literature) if $K$ is $l_{2}$-deficient. It should be noted that by Schori [8] homeomorphisms of $M$ onto $M \times F$ do exist. The main result of this paper is that all four definitions are equivalent.

\section{The main theorems.}

THEOREM 2.1. Let $K$ be a closed subset of an F-manifold $M$, where $F$ is a $M L C T V S$ such that $F \cong F^{\omega}$. Then the following are equivalent:

(1) $K$ is locally a subset of a collared submanifold of $M$,

(2) $K$ is locally infinite-deficient,

(3) $K$ is locally F-deficient,

(4) $K$ is F-deficient,

(5) $K$ is infinite-deficient,

(6) $K$ is the finite union of sets each of which satisfies one of the above.

Proof. Klee was the first to show that $l_{2} \times[0,1)$ is homeomorphic to $l_{2}$ (Theorem III (1.3) of [6]). Since $F$ is locally convex, it is known that $F \cong R \times Z$ where $Z$ is some metric space. Using $F \cong F^{\omega}$ it follows easily that $F \cong F \times l_{2}$. This combined with the first statement shows that $(2) \Rightarrow(1)$. Again using $F \cong F \times l_{2}$, it follows that (4) $\Rightarrow(5)$ and (3) $\Rightarrow(2)$, for if $(K, M) \subset$ $(\{0\} \times M, F \times M)$, then $(K, M) \subset\left(\{0\} \times\{0\} \times M, l_{2} \times F \times M\right) \subset(\{0\} \times F \times M$, $\left.l_{2} \times F \times M\right)=\left(\{0\} \times M, l_{2} \times M\right)$. Also (5) $\Rightarrow(2)$ and $(4) \Rightarrow(3)$ are obvious. That $(1) \Rightarrow(3)$ follows from Proposition 5.1 of $\S 5$. Finally, Proposition 5.3 of $\S 5$ shows that $(6) \Rightarrow(4)$ when the sets in the finite union satisfy property (3).

THEOREM 2.2. Let $F$ and $M$ satisfy the hypotheses of Theorem 2.1, and let $K$ satisfy any of the equivalent conditions in the theorem. Then there is a homeomorphism $h: M \rightarrow M \times F$ such that for $x \in K, h(x)=(x, 0)$.

Proof. Apply Theorem 2.1 and Proposition 5.3.

3. Definitions. By the cone over $X$, we shall mean $X \times I$ with the points $X \times\{0\}$ identified, with the following topology: At the vertex, a basis for the open sets will be the sets $\{(x, t) \in X \times I \mid t<s\}$ for each $s \in(0,1)$, and at other points the product topology will be used. The open cone, denoted $C(X)$, will be the cone minus $X \times\{1\}$.

By Lemma 2 of [5], $F \cong C(E)$ for some metric space $E$. Let $h: F \rightarrow C(E)$ be such a homeomorphism. For $y \in F$, define $\|y\|=\pi_{2} h(y)$, that is, the second coordinate of $h(y)$. If $\|y\|=0$, we call $y$ the 0 -element in $F$. Let $B_{t}=$ $\{y \in F \mid\|y\|<t\}$. 
Let $X$ be a topological space, $F$ a MLCTVS, and $r: X \rightarrow I$ a continuous function. Then the variable product of $X$ by $F$ with respect to $r$ is defined to be

$$
X \times{ }_{r} F=\{(x, y) \in X \times F \mid y=0 \text { or }\|y\|<r(x)\} .
$$

We say that this variable product is zero over $r^{-1}(0)$. For $U$ a subset of $X$, the fiber over $U$ is the set $(U \times F) \cap\left(X \times{ }_{r} F\right)$, and may also be called the variable product restricted to $U$.

4. Isotopy lemmas. The following technical lemmas construct isotopies which prove to be quite useful in pushing around deficient sets. All of the isotopies will be invertible (have continuous inverses).

LEMMA 4.1 (SCHORI). There exists an isotopy $h_{t}: F \times F \rightarrow F \times F, t \in I$, satisfying

(1) $h_{0}=\mathrm{id}$,

(2) for $t<1, h_{t}$ is onto $B_{(1-t)} \times F$,

(3) $h_{1}$ is onto $\{0\} \times F$.

The proof is due to Anderson and Schori (see Lemma 5.3 of [8]).

Lemma 4.2. There exists a double isotopy ${ }_{r} f_{t}: F \rightarrow F, r \in(0,1], t \in$ $[0,1+r / 2]$ satisfying

(1) ${ }_{r} f_{0}=\mathrm{id}$,

(2) for $t<1,{ }_{r} f_{t}$ is a homeomorphism,

(3) ${ }_{r} f_{1}$ is onto $F-\{0\}$,

(4) for $t>1,{ }_{r} f_{t}$ is onto $f-\bar{B}_{(t-1)}$,

(5) ${ }_{r} f_{t}$ is the identity outside $B_{r}$.

Proof. For $t \leqq 1$, the isotopy is constructed using the isotopy pushing the origin off $l_{2}$ which is defined by Anderson and Bing [2, pp. 784-786] and the fact that $F$ has $l_{2}$ as a topological factor. For $t \geqq 1$, the isotopy is easily constructed using $\|y\|$ for $y \in F$.

LEMmA 4.3. There exists a double isotopy ${ }_{r} g_{t}: F \times F \rightarrow F \times F, r \in(0,1]$, $t \in I$, satisfying

(1) ${ }_{r} g_{0}=\mathrm{id}$,

(2) for $t<1,{ }_{r} g_{t}$ is onto a variable product of $F$ by $F$ which is nowhere zero,

(3) ${ }_{r} g_{1}$ is onto a variable product of $F$ by $F$ which is zero over $\{0\}$,

(4) ${ }_{r} g_{t}=\mathrm{id}$ outside $B_{r} \times F$ and on $F \times\{0\}$.

Proof. Using the isotopy of Lemma 4.2, define

$$
{ }_{r} g_{t}(x, y)=\left({ }_{r_{1}} f_{t_{1}}(x), y\right)
$$


where $r_{1}$ and $t_{1}$ are functions defined by

$$
\begin{aligned}
r_{1} & =\min \{r,\|y\|\}, & & \\
t_{1} & =0 & & \text { for } y=0, \\
& =(1+r / 2)-r / 2 t(\|y\|+1) & & \text { for }\|y\| \geqq(1-t) / t, \\
& =\max \{0,\|y\|+1-(1-t) / t\} & & \text { for }\|y\| \leqq(1-t) / t,(1-t) / t \geqq 1, \\
& =t\|y\| /(1-t) & & \text { for }\|y\| \leqq(1-t) / t \leqq 1 .
\end{aligned}
$$

\section{Three propositions.}

Proposition 5.1. $([0,1) \times F,\{0\} \times F)$ and $(F \times F,\{0\} \times F)$ are homeomorphic as pairs.

Proof. Regard the $F$ in the first pair as being $F \times F$. Using the isotopy of Lemma 4.1 , define $f:[0,1) \times F \times F \rightarrow[0,1) \times F \times F$ by $f(s, x, y)=$ $\left(s, h_{(1-s)}(x, y)\right)$.

It is clear that $f$ is an embedding onto a variable product of $[0,1) \times F$ by $F$ which is zero over $\{0\} \times F$, and this variable product is easily seen to be homeomorphic to the open cone of $F$ crossed with $F$. Furthermore, $f$ takes the set $\{0\} \times F \times F$ onto the vertex of the cone crossed with $F$. Since $F$ is homeomorphic to its unit sphere (use $F \cong(0,1) \times F$ to show that the unit sphere is homeomorphic to $F-\{0\}$ and fill in the origin using Lemma 4.2), it follows that the open cone over $F$ is homeomorphic to $F$.

Proposition 5.2. Let $M$ be an $F$-manifold, and let $K$ be a closed locally $F$-deficient subset of $M$. Let $p: M \rightarrow(0,1]$ be a continuous function. Then there is a continuous function $r: M \rightarrow I$ and a homeomorphism $h: M \times F \rightarrow$ $M \times{ }_{r} F$ such that $r^{-1}(0)=K, h=\mathrm{id}$ on $M \times\{0\}$, and for $(x, y) \in M \times F$, $d\left(x, \pi_{1} h((x, y))\right)<p(x)$, where $d$ is the metric on $M$.

The proposition does not follow from [8], as might be suspected. The condition " $h=\mathrm{id}$ on $M \times\{0\}$ " is crucial. The proof makes use of "lateral" pushes rather than "vertical" ones as in [8].

Proof. We use a theorem due to E. Michael [7] which is quite useful.

THEOREM (MICHAEL). Let $\mathbf{P}$ be a topological property satisfying

(a) if $U$ has Property $\mathrm{P}$, then every open subset of $U$ has Property $\mathrm{P}$,

(b) if $U$ is the union of two open (in $U$ ) sets both of which have Property $\mathrm{P}$, then $U$ has Property $\mathrm{P}$,

(c) if $U$ is the union of disjoint open (in $U$ ) sets, each of which has Property $\mathrm{P}$, then $U$ has Property $\mathrm{P}$.

If $X$ is a paracompact Hausdorff space having Property $\mathrm{P}$ locally, then $X$ has Property $\mathrm{P}$.

Definition. An open subset $U$ of $M$ has Property $\mathrm{P}$ if given $W$ an open set in $M, C$ and $D$ closed subsets of $K$, and $p: M \rightarrow(0,1]$ and $r_{0}: M \rightarrow I$ 
continuous functions such that $C \subset W \subset U$ and $r_{0}^{-1}(0)=D$; then there is a continuous function $r_{1}: M \rightarrow I$ and a homeomorphism $h: M \times{ }_{r_{0}} F \rightarrow M \times{ }_{r_{1}} F$ such that

(1) $r_{1} \leqq r_{0}$ and $r_{1}^{-1}(0)=D \cup C$,

(2) $h \mid M \times\{0\}=$ id

(3) $h \mid(M-W) \times{ }_{r_{0}} F=\mathrm{id}$,

(4) for $(x, y) \in M \times{ }_{r_{0}} F, d\left(x, \pi_{1} h((x, y))\right)<p(x)$.

That Property P satisfies conditions (a) and (c) of Michael's theorem is easy to check. As is always the case, condition (b) is more complicated to show.

Let $U_{1}$ and $U_{2}$ be open subsets of $M$ having Property $\mathrm{P}$ and let $U=$ $U_{1} \cup U_{2}$. Let $C, W, D, p$ and $r_{0}$ be as in the definition of Property P. For $i=1,2$, pick open sets $U_{i}^{\prime}$ and $U_{i}^{\prime \prime}$ such that $\bar{U}_{i}^{\prime \prime} \subset U_{i}^{\prime}$ and $\bar{U}_{i}^{\prime} \subset U_{i}$ and $U_{1}^{\prime \prime} \cup$ $U_{2}^{\prime \prime}=U$. For $i=1,2$, let $W_{i}=U_{i}^{\prime} \cap W$ and $C_{i}=\bar{U}_{i}^{\prime \prime} \cap C$. Let $D_{1}=D$ and $D_{2}=$ $D \cup C_{1}$. Define $p^{*}: M \rightarrow(0,1]$ by

$$
p^{*}(x)=\frac{1}{3} \max \left\{\delta \in(0,1] \mid p(z) \geqq \delta \text { for all } z \in N_{\delta}(x)\right\},
$$

where $N_{\delta}(x)$ is the $\delta$-neighborhood of $x$.

Since $U_{1}$ has Property $\mathrm{P}$, there exist a continuous function $r_{1}: M \rightarrow I$ and a homeomorphism $h_{1}: M \times{ }_{r_{0}} F \rightarrow M \times{ }_{r_{1}} F$ satisfying (1) $r_{1} \leqq r_{0}$ and $r_{1}^{-1}(0)=$ $D_{1} \cup C_{1}$, (2) $h_{1} \mid M \times\{0\}=\mathrm{id}$, (3) $h_{1} \mid\left(M-W_{1}\right) \times_{r_{0}} F=\mathrm{id}$, (4) for $(x, y) \in$ $M \times{ }_{r_{0}} F, d\left(x, \pi_{1} h_{1}((x, y))\right)<p^{*}(x)$.

Similarly, since $U_{2}$ has Property $\mathrm{P}$, there exist a continuous function $r_{2}: M \rightarrow I$ and a homeomorphism $h_{2}: M \times{ }_{r_{1}} F \rightarrow M \times{ }_{r_{2}} F$ satisfying (1) $r_{2} \leqq r_{1}$ and $r_{2}^{-1}(0)=D_{2} \cup C_{2}$, (2) $h_{2} \mid M \times\{0\}=\mathrm{id}$, (3) $h_{2} \mid\left(M-W_{2}\right) \times{ }_{r_{1}} F=\mathrm{id}$, (4) for $(x, y) \in M \times{ }_{r_{1}} F, d\left(x, \pi_{1} h_{2}((x, y))\right)<p^{*}(x)$.

Then the functions $r_{2}: M \rightarrow I$ and $h_{2} * h_{1}: M \times{ }_{r_{0}} F \rightarrow M \times{ }_{r_{2}} F$ satisfy the requirements in the definition of Property $P$.

If we could show that $M$ has Property P, we would be finished, for we could let $C=W=U=M$ and $r_{0}(m)=1$. Hence it suffices by Michael's theorem to show that $M$ has Property $\mathrm{P}$ locally. It is sufficient to show this when $U=F \times F$ and $K \cap U \subset F \times\{0\}$. Let $C, W, D, p$ and $r_{0}$ be as before. Let $F_{1}=F \times\{0\}$. Let $r: F_{1}-D \rightarrow I$ be defined by

$$
r(x)=\min \left\{d((x, 0), W-D), p^{*}(x)\right\} .
$$

Let $t: F_{1}-D \rightarrow I$ be a continuous function such that $t^{-1}(1)=C-D$ and $t^{-1}(0) \supset\left(F_{1}-D\right)-W$. Define $f:\left(F_{1}-D\right) \times F \times F \rightarrow\left(F_{1}-D\right) \times F \times F$ by

$$
\begin{aligned}
f(x, y, z) & =\left(x,{ }_{r(x)} g_{t(x)}(y, z)\right) & & \text { if } r(x) \neq 0, \\
& =\text { id } & & \text { if } r(x)=0,
\end{aligned}
$$

where $g$ is the double isotopy of Lemma 4.3. 
It is easily seen that $f$ is a homeomorphism onto a variable product of $\left(F_{1}-D\right) \times F$ by $F$ which is zero on $C-D$. Since the variable product of $\left(F_{1}-D\right) \times F$ by $F$ with respect to $r_{0}$ is nowhere zero, we can regard this product as $\left(F_{1}-D\right) \times F \times F$, and applying $f$ to this and extending by the identity to $(D \times F) \times{ }_{r_{0}} F$, we will get a variable product and a homeomorphism satisfying the requirements in the definition of Property $P$.

Proposition 5.3. Let $M$ be an F-manifold, and let $K$ be a closed subset of $M$ which is the finite union of closed locally $F$-deficient sets. Then there is a homeomorphism $h: M \rightarrow M \times F$ such that for $x \in K, h(x)=(x, 0)$.

We may assume that $K=\bigcup_{1}^{n} K_{i}$ where each $K_{i}$ is closed and locally $F$ deficient. Using Proposition 5.2, there is a homeomorphism of $M \times F$ onto a variable product of $M$ by $F$ which is zero on $K_{1}$. Since this variable product restricted to $M-K_{1}$ is nonzero, we can regard the variable product over $M-K_{1}$ as just $\left(M-K_{1}\right) \times F$, and can apply Proposition 5.2 with the function $p$ as some continuous function approaching 0 at $K_{1}$, and we will get a homeomorphism onto a variable product which is zero on $K_{2}-K_{1}$. Using the function $p$, one can show that this latter homeomorphism can be extended continuously by the identity to the set $K_{1} \times\{0\}$. Then the composition of the two homeomorphisms gives a homeomorphism from $M \times F$ onto a variable product of $M$ by $F$ which is the identity on $M \times\{0\}$ and is zero on $K_{1} \cup K_{2}$.

Repeated applications of Proposition 5.2 result in a homeomorphism of $M \times F$ onto a variable product of $M$ by $F$ which is zero over $K$ and is the identity on $M \times\{0\}$. Using the stability theorem of Schori (Corollary 2.3 of [8]) there is a homeomorphism $h:(M-K) \times F \rightarrow(M-K)$ satisfying

$$
d(x, h((x, y)))<d(x, K) / 2 \text { for }(x, y) \in(M-K) \times F .
$$

Applying this homeomorphism to the variable product over $M-K$ (which, since it is nowhere zero, can be regarded as $(M-K) \times F)$, one can extend continuously by the identity to $K \times\{0\}$, and the proof is completed.

\section{BIBLIOGRAPHY}

1. R. D. Anderson, On topological infinite deficiency, Michigan Math. J. 14 (1967), 365-383. MR 35 \#4893.

2. R. D. Anderson and R. H. Bing, A complete elementary proof that Hilbert space is homeomorphic to the countable infinite product of lines, Bull. Amer. Math. Soc. 74 (1968), 771-792. MR 37 \#5847.

3. T. A. Chapman, Deficiency in infinite-dimensional manifolds, Gen. Topology Appl. 1. (1971), 263-272.

4. W. H. Cutler, Negligible subsets of infinite-dimensional Fréchet manifolds, Proc. Amer. Math. Soc. 23 (1969), 668-675. MR 40 \#2133. 
5. D. W. Henderson, Corrections and extensions of two papers about infinite-dimensional manifolds, Topology (to appear).

6. V. L. Klee, Jr., Convex bodies and periodic homeomorphisms in Hilbert space, Trans. Amer. Math. Soc. 74 (1953), 10-43. MR 14, 989.

7. E. A. Michael, Local properties of topological spaces, Duke Math. J. 21 (1954), 163-171. MR 15, 977.

8. R. Schori, Topological stability for infinite-dimensional manifolds, Compositio Math. 23 (1971), 87-100.

Department of Mathematics, Louisiana State University, Baton Rouge, LOUISIANA 70803 